\title{
Kernel Fisher for Shape Based Classification in Epilepsy
}

\author{
N. Vohra ${ }^{1}$, B. C. Vemuri ${ }^{1}$, A. Rangarajan ${ }^{1}$, R.L. Gilmore ${ }^{2}$, \\ S.N. Roper ${ }^{3}$, and C. M. Leonard ${ }^{4}$ \\ 1 CISE Department, University of Florida, Gainesville, FL 32601, USA \\ 2 Dept. of Neurology, University of Florida, Gainesville, FL 32601, USA \\ 3 Dept. of Neurosurgery, University of Florida, Gainesville, FL 32601, USA \\ 4 Dept. of Neuroscience, University of Florida, Gainesville, FL 32601, USA
}

\begin{abstract}
In this paper, we present the application of Kernel Fisher in the statistical analysis of shape deformations that might indicate the hemispheric location of an epileptic focus. The scans of two classes of patients with epilepsy, those with a right and those with a left medial temporal lobe focus (RATL and LATL), as validated by clinical consensus and subsequent surgery, were compared to a set of age and sex matched healthy volunteers using both volume and shape based features. Shape based features are derived from the displacement field between the left and right hippocampii of a healthy subject/patient. The results show a significant improvement in distinguishing between the controls and the rest (RATL and LATL) using only the shape as opposed to volume based features. We also achieve a reasonable improvement in the efficiency to distinguish between RATL and LATL based on shape in comparison to volume information. It should be noted that automated identification of hemispherical foci of epilepsy has not been previously reported.
\end{abstract}

\section{Introduction}

Statistical analysis of shape deformations, such as those likely to occur in epilepsy and other neurological disorders, necessitate both global and local parameter based characterization of the object under study. The most popular method to achieve the same has been size and volume based analysis. However, this captures only one of the aspects necessary for complete characterization while shape based analysis gives much more information, which can be combined with the former to help understand the anatomical structures better.

In this paper, we focus on developing an automatic technique which can aid in distinguishing between controls and patients with epilepsy and can indicate the hemispheric location of an epileptic focus (right medial temporal lobe or left medial temporal lobe) in the patients. It should be noted that the work does not attempt to determine the precise coordinates of the epilepsy focus in the patients.

\subsection{Literature Review}

To use the classification techniques such as Support Vector Machines and Fisher Discriminant for solving the problem of statistical analysis of anatomical shape

T. Dohi and R. Kikinis (Eds.): MICCAI 2002, LNCS 2489, pp. 436-443 2002.

(C) Springer-Verlag Berlin Heidelberg 2002 
differences between different populations has been the focus of various researchers in recent past. The choice of feature vectors to capture maximum information plays an important part of the study.

Gerig and Styner 3] proposed the use of both volume measurements and shape based features (Mean Square Distance) to detect group differences in hippocampal shape in schizophrenia. The class differences are then accounted for by using SVM followed by performance evaluation using the leave-one-out technique. From the results reported, it can be concluded that shape alone could not capture the class differences. This failure can be attributed either to weak shape features or the fact that the natures of the groups under study is such that shape alone cannot represent the entire class character. Joshi et al. 4] used high dimensional transformations of a brain template to compare the hippocampal volume and shape characteristics in schizophrenia and control subjects. Linear discriminant analysis was used to measure the performance of the selected feature vector. Use of fluid flow model is computationally expensive. Recently, Golland et al. [5] have studied hippocampal shape differences between schizophrenia patients and normal controls. The shapes were represented using implicit functions and classified using an SVM with a Gaussian kernel. Marginal increases over volume based methods were reported using this technique.

\subsection{Overview of Our Algorithm}

In this paper, we demonstrate the application of the kernel Fisher algorithm for shape based classification of hippocampal shapes in controls and epilepsy. Given a pair of sparse sets of data points corresponding to the outlines of the left and right hippocampii of a subject, appropriate shape features are extracted by first fitting a model to the data sets using a deformable pedal surface [2]. This is followed by a rigid and a non-rigid registration of the left and right hippocampii using the Iterative Closest Point algorithm [1] and level-set method [7] respectively. The local deformations obtained by non-rigid registration are then fed into the kernel fisher classifier to capture the statistical difference between the three known classes in epilepsy. The choice of kernel Fisher as the classifier has been motivated by the fact that it can separate the classes in a very high or infinite dimensional space using a linear classier and is simple to implement. The kernel Fisher training algorithm used in this work does not require non-linear optimization like SVM and hence is computationally more efficient. Note that the optimization that the SVM tries to solve is a quadratic programming problem with constraints and is known to be NP-complete. Kernel Fisher has shown results comparable to SVM in various other applications 6].

\subsection{Organization of the Paper}

The rest of the paper is organized as follows: Section 2 describes the snake pedal model used for fitting a model to given data points followed by the procedure of selection of shape based features. In Section 3, we summarize the kernel Fisher method as a classifier. Sections 4 presents the experimental results followed by conclusions in Section 5 


\section{Shape Extraction and Features}

In this section we will discuss the schemes used to segment the region of interest followed by the methods employed for rigid and non-rigid registration of the corresponding hippocampii for a given subject.

\subsection{Overview of the Shape Modeling Scheme}

In order to segment the region of interest in the given image, we use a deformable pedal surface described in [2. Pedal curves/surfaces are defined as the loci of the foot of the perpendiculars to the tangents of a fixed curves/surface from a fixed point called the pedal point 2. A large class of shapes can be synthesized by varying the position of the pedal point which exhibits both global and local deformations. Physics-based control is introduced by using a snake to represent the position of this varying pedal point. Thus the model is called as "snake pedal" and allows for interactive manipulation through forces applied to the snake. The model also allows representation of global deformations such as bending and twisting without introducing additional parameters. To fit a model to a given set of data points in 2D/3D, a non-linear optimization scheme using LevenbergMarquardt (LM) method in outer loop for estimating global parameters and the Alternating Direction Implicit (ADI) method in the inner loop for estimating the local parameters of the model is employed 2 .

\subsection{Shape Registration}

Shape registration, in general, is required at both global and local levels. In the present work we use the Iterative Closest Point algorithm proposed in [1] to determine the rotation and translation between a subject's left and right hippocampus. The choice of ICP algorithm is motivated by the fact that snake-pedal based model fitting yields an extrinsic parameterization which is not suitable for use in finding the corresponding points between the left and right hippocampii. The corresponding left and right hippocampus of a subject may have a global scaling factor which is accounted for by approximating the shapes of the smallest ellipsoid that encloses the hippocampus and then equalizing their corresponding eigen values. The problem of finding the non-rigid estimation can be formulated as a motion estimation task, in particular, estimation of the displacement field between the two given shapes. We use the level-set formulation described in [7] to estimate the displacement field that leads to the following governing equation:

$$
\begin{gathered}
\overrightarrow{V_{t}}=\left[d_{2}(X)-d_{1}(\vec{V}(X))\right] \frac{\Delta\left(G_{\sigma} * D_{1}(\vec{V}(X))\right)}{\left\|\Delta\left(G_{\sigma} * D_{1}(\vec{V}(X))\right)\right\|+\alpha}+\lambda\left(\begin{array}{c}
\Delta u \\
\Delta v \\
\Delta w
\end{array}\right) \\
\text { with } \vec{V}(X, 0)=\overrightarrow{0}
\end{gathered}
$$

where $d_{1}$ and $d_{2}$ denote the signed distance images of the source and target shapes, $\Delta$ denotes the Laplacian operator, $G_{\sigma}$ is Gaussian kernel and $\alpha$ is a small 
positive number called as stabilizing factor. The above differential equation can be solved by using the numerical implementation described in [7. Note that the signed distance images can be obtained by using the Fast Marching Method (FMM) described in Sethian [8].

\section{Kernel Fisher}

The classification problem can then be approached in two ways, namely supervised and unsupervised, with the discriminant function being linear or non-linear. The classical approach begins with the optimal Bayes classifier by assuming the normal distribution for the classes which using the linear discriminant analysis leads to the Fisher algorithm. The Fisher approach is based on projecting d-dimensional data onto a line with the hope that the projections are well separated by class. Thus, the line is oriented to maximize this class separation.

However, the features in the input space may not possess sufficient discriminatory power for separation of class via linear projection techniques. This problem can be tackled by mapping the input data into a very high dimensional space and using a linear classifier in this new feature space thereby giving an implicit non-linear classification in the input space. This is the basic idea behind the kernel Fisher algorithm. Let $\phi$ be a non-linear mapping to some feature space $\mathcal{F}$. Thus, separation in the new feature space can be found by maximizing:

$$
J(\underline{w})=\frac{\underline{w}^{T} S_{B}^{\phi} \underline{w}}{\underline{w}^{T} S_{W}^{\phi} \underline{w}}
$$

where $\underline{w} \in \mathcal{F}, S_{B}^{\phi}$ and $S_{W}^{\phi}$ are defined as follows

$$
S_{B}^{\phi}=\left(m_{1}^{\phi}-m_{2}^{\phi}\right)\left(m_{1}^{\phi}-m_{2}^{\phi}\right)^{T}, \quad S_{W}^{\phi}=\sum_{i=1,2} \sum_{x \in \chi_{i}}\left(\phi(x)-m_{i}^{\phi}\right)\left(\phi(x)-m_{i}^{\phi}\right)^{T}(3)
$$

with $m_{i}^{\phi}=\frac{1}{l_{i}} \sum_{j=1}^{l_{i}} \phi\left(x_{j}^{i}\right)$.

Eqn. (2) can be solved by formulating it in terms of dot-products $(\phi(x) \cdot \phi(y))$ of the training patterns [6] which can then be evaluated using Mercer kernels $(k(\mathbf{x}, \mathbf{y})=(\phi(\mathbf{x}) \cdot \phi(\mathbf{y})))$ [6]. As explained in [6] using the kernel theory, (2) can be rewritten as

$$
J(\alpha)=\frac{\alpha^{T} M \alpha}{\alpha^{T} N \alpha}
$$

where

$$
\begin{gathered}
M=\left(M_{1}-M_{2}\right)\left(M_{1}-M_{2}\right)^{T} \text { with }\left(M_{i}\right)_{j}=\frac{1}{l_{i}} \sum_{k=1}^{l_{i}} k\left(x_{j}, x_{k}^{i}\right) \\
N=\sum_{j=1,2} K_{j}\left(I-1_{l_{j}}\right) K_{j}^{T}, \quad K_{j}: l \times l_{j} \text { matrix s.t. }\left(K_{j}\right)_{n m}=k\left(x_{n}, x_{m}^{j}\right) \\
I: \text { identity matrix }, 1_{l_{j}}: l_{j} \times l_{j} \text { matrix with all entries } 1 / l_{j}
\end{gathered}
$$


and $\alpha$ are the coefficients corresponding to the training patterns s.t. $\underline{w}=$ $\sum_{i=1}^{l} \alpha_{i} \phi\left(x_{i}\right)[6]$.

The optimum direction of projection can be found by taking the leading eigenvector of $N^{-1} M$. This approach is called as Kernel Fisher Discriminant (KFD) [6]. The projections of a new vector $\underline{x}$ onto $\underline{w}$ can be obtained by

$$
(\underline{w} \cdot \phi(x))=\sum_{i=1}^{l} \alpha_{i} k\left(x_{i}, x\right)
$$

The proposed setting is ill-posed due to the estimation of $l$-dimensional covariance structures from $l$ samples which can cause the matrix $N$ to be nonpositive [6]. The problem can be solved by adding a multiple identity matrix to $N$ [6] such that

$$
N_{\mu}=N+\mu I
$$

\section{Experimental Results and Validation}

In this section, we present the experimental results obtained by testing the performance of Linear Fisher and kernel Fisher on hippocampal shapes of 25 control subjects, 11 LATL and 14 RATL patients. Given the points sets for the left and right hippocampii of a healthy subject/patient, we begin with model fitting using the Snake Pedal Model. The superimposed mesh of 21 x 40 on the model gives a new point set of size $840 \times 3$ (each point is in $3 \mathrm{D}$ ). The fitted point sets are first registered globally using ICP algorithm and ellipsoid based technique. This is followed by local registration using level-set method which uses signed distance images of size 128 x 128 x 128 obtained by the Fast Marching method.

The displacement field obtained for the fitted point sets is then used to form two types of shape based features. The first type is called sign of displacement and and the other is called direction of the displacement vector. The sign of the displacement is defined as follows. Given the displacement vector for a point on the zero-set of the source image, determine the cube in which the displaced point falls in the source image. Depending on the sign of the vertices (since each vertex was assigned +/- sign while forming the distance image) of the enclosing cube, assign a sign to the magnitude of the displacement. The direction vector is obtained by finding the unit vector corresponding to the displacement vector at each point on the zero set. We have chosen this as our feature vector since we believe that the displacement vector direction allows us to capture the differences between the two classes. However, the issue of including the magnitude information of the displacement field at each point on the zero set is an important one and we hope to investigate it in future work. The feature vector for the sign of displacement is of length 762 while the direction vector is of length $762 \times 3$ (each point has $\mathrm{x}, \mathrm{y}, \mathrm{z}$ component of displacement). These numbers are derived from the fact that there are 840 points on the zero set and the first and last row of the 21 x 40 mesh represents the north and south pole as described in [2]. In the present 
study, we did not include feature pruning. Since the feature vector dimensionality far exceeds the number of retrospective patient studies, feature selection and pruning using principal component analysis (PCA) or related strategies can play an important role in improving the generalization performance. Our preliminary forays into feature pruning are very promising and we plan to vigorously pursue this line of investigation in future work. The shape based results are also compared to the ones obtained by using the volume information only with $\mathrm{L} / \mathrm{R}$ and $(\mathrm{L}-\mathrm{R}) /(\mathrm{L}+\mathrm{R})$ as the feature vector where $\mathrm{L}$ and $\mathrm{R}$ are the volume of left and right hippocampii.

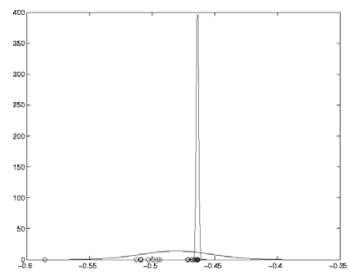

(a) Linear Fisher

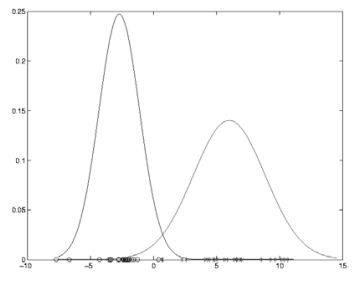

(b) Linear Fisher

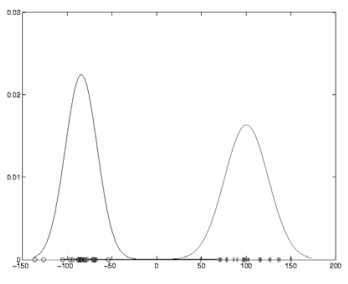

(c) Kernel Fisher

Fig. 1. CTRL vs Rest (a) Fvec: Volume based, (b) Fvec: Sign of displacement, (c) Fvec: Sign of displacement

Table 1. Controls vs Rest (Controls $=24$, Rest $=25)$

\begin{tabular}{|l||l|l||l|l||l|l|}
\hline \multicolumn{2}{|l|}{} & Volume & \multicolumn{2}{l||}{ Sign of Displacement } & Direction Vector \\
\hline Classifer & Training & Testing & Training & Testing & Training & Testing \\
\hline \hline Linear Fisher & $64.43 \%$ & $61.22 \%$ & $95.92 \%$ & $87.76 \%$ & $96.5 \%$ & $85.71 \%$ \\
\hline KF-Poly $(\mathrm{d}=2)$ & $55.2 \%$ & $55 \%$ & $100 \%$ & $91.84 \%$ & $100 \%$ & $95.9 \%$ \\
\hline KF-RBF & $64.93 \%$ & $61.22 \%$ & $100 \%$ & $89.98 \%$ & $100 \%$ & $93.8 \%$ \\
\hline
\end{tabular}

Fig. 1 shows the results of the Linear Fisher and Kernel Fisher (using radial basis function as the kernel) with a feature vector based on volume and shape for controls vs patients. It can be seen from Fig. 1a that using just the volume information does not distinguish between the subjects who need surgery and who do not. Fig. 1b and Fig. 1c show considerable improvement with a feature vector as sign of displacement in particular with Kernel Fisher as the classifier. Plots using direction vector are similar to the ones obtained using sign of displacement. Table 1 summarizes the training set accuracy and the cross-validation accuracy using leave-one-out for the feature vectors and classifiers considered.

Given the classification between controls and patients, the next task is to identify the side of focus. This is again done by comparing the shape features for RATL and LATL. Fig. 2 shows the separation between the two classes using volume and shape features. It is clear that volume (Fig. 2a) cannot distinguish between them easily. The sign of displacement using linear Fisher (Fig. 2b) also does not show a good separation. However, kernel Fisher with shape features 
(Fig. 2c) is able to capture it much better. Table 2 summarizes the training and leave-one-out accuracy for RATL and LATL using volume and shape features.

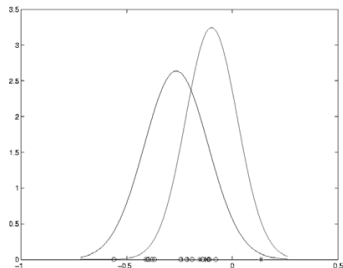

(a) Linear Fisher

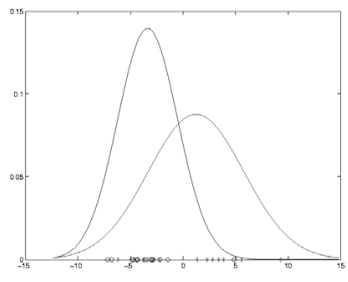

(b) Linear Fisher

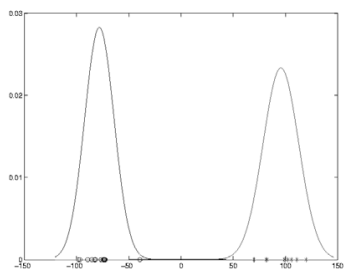

(c) Kernel Fisher

Fig. 2. LATL vs RATL. (a) Fvec: Volume based, (b) Fvec: Sign of displacement, (c) Fvec: Sign of displacement

Table 2. LATL vs RATL $(\mathrm{LATL}=11$, RATL $=14)$

\begin{tabular}{|l||l|l||l|l||l|l|}
\hline \multicolumn{2}{|c|}{} & Volume & \multicolumn{2}{l||}{ Sign of Displacement } & Direction Vector \\
\hline Classifer & Training & Testing & Training & Testing & Training & Testing \\
\hline \hline Linear Fisher & $66.88 \%$ & $64 \%$ & $74.88 \%$ & $64 \%$ & $88 \%$ & $68 \%$ \\
\hline KF-Poly $(\mathrm{d}=2)$ & $66.88 \%$ & $64 \%$ & $100 \%$ & $72 \%$ & $100 \%$ & $72 \%$ \\
\hline KF-RBF & $66.88 \%$ & $64 \%$ & $100 \%$ & $72 \%$ & $100 \%$ & $68 \%$ \\
\hline
\end{tabular}

It can be seen that we are able to distinguish between controls and patients with a much higher accuracy as compared to distinguishing between RATL and LATL. This can be due to various reasons. The shape differences among the pathologies may be highly correlated, hence making it difficult to separate them out. Also the number of data samples for the patients with pathology is quite small which hinders a sufficient representation of the population. This can also be seen in high training accuracy but low test accuracy among patients.

\section{Discussion and Conclusion}

Our entire approach is predicated on using shape-based features for discriminating between normal controls and subjects diagnosed with epilepsy as well as indicating the hemispheric location of epileptic focus in the patients. It should be noted that the work does not attempt to determine the precise coordinates of the focus of epilepsy in the patients. Since the feature vectors may not be linearly separable, we embarked upon a kernel Fisher strategy in which the patterns are first mapped to an infinite dimensional space before computing the Fisher discriminant. The choice of kernel is crucial for achieving good generalization. This issue requires much more empirical testing and validation in order to determine the best kernel for the task. Unfortunately, the deeper and more fundamental relationship between the feature vector density function and the 
choice of the kernel mapping function cannot be empirically explored due to data being available only for a small number of subjects.

The choice of feature vector is key to achieving good training and generalization performance. We have shown that the sign of the displacement vector can capture some of the shape differences between the two classes of subjects. Based on our empirical results, we conclude that control subjects and subjects of pathology can be discriminated using shape features. However, the same shape features are less successful in inter-hemispheric discrimination between subjects of pathology. We expect to improve the classification performance in this area by i) increasing the number of patient studies, ii) better feature selection and pruning and iii) improving the classifier.

\section{Acknowledgment}

This research was in part funded by the NSF grant IIS-9811042 and NIH RO1RR13197.

\section{References}

1. Besl P.J., McKay N.D.: A Method of Registration of 3-D Shapes. IEEE Trans. of Pattern Analysis and Machine Intelligence, Vol. 14, No. 2 (1992) 239-255

2. Vemuri B.C., Guo Y.: Snake Pedals: Compact and Versatile Geometric Models with Physics-based Control. IEEE Trans. on Pattern Analysis and Machine Intelligence, Vol 22, No. 5 (2000) 445-459

3. Gerig G., Styner M., Shenton M.E., Lieberman J.A.: Shape vs Size: Improved understanding of the Morphology of Brain Structures. MICCAI, (2001) 24-32

4. Csernansky J.G., Joshi S., Wang L., Haller J.W., Gado M., Miller J.P., Grenander U., Miller M.I.: Hippocampal morphometry in schizophrenia by high dimensional brain mapping. Neurobiology, Vol. 95, Issue 19 (1998) 11406-11411

5. Golland P., Grimson W.E.L, Shenton M.E., Kikinis R.: Small Sample Size Learning for Shape Analysis of Anatomical Structures. MICCAI, LNCS 1935 (2000) 72-82

6. Mika S., Rätsch G., Weston J.: Fischer Discriminant Analysis with Kernels. Neural Networks for Signal Processing IX, IEEE (1999) 41-48

7. Vemuri B., Ye J., Chen Y., Leonard C.: A Level-set based Approach to Image Registration. Workshop on Mathematical Methods in Biomedical Image Analysis, June11-12 (2000) 86-93

8. Sethian J.A.: Level Set Methods and Fast Marching Methods:Evolving Interfaces in Computational Geometry, Fluid Mechanics, Computer Vision and Material Science. Cambridge University Press (1999) 